Haben Sie Fragen zur Abrechnung oder zur wirtschaftlichen Praxisführung? Als Leser der MMW können Sie sich an unseren Experten wenden: Helmut Walbert, Facharzt für Allgemeinmedizin und Betriebswirt aus Würzburg.

\title{
Unterlagen haben Sie zehn Jahre lang am Hals
}

\section{a Dr. B. Pflug, Allgemeinärztin, Bran- denburg: Wie lange müssen wir Patientenunterlagen aufbewahren?}

MMW-Experte Walbert: Zehn Jahre ab dem Tag der letzten Behandlung bzw. des letzten Kontakts zum Patienten. Das gilt grundsätzlich für alle den $\mathrm{Pa}$ tienten betreffende Unterlagen vom Erstkontakt an. Wird eine Praxis übernommen, ohne dass übergangsweise ge meinsam gearbeitet wird, müssen die Patientenakten des Vorgängers erst einmal unter Verschluss. Erst wenn der Patient den neuen Arzt aufsucht, stimmt er formal der Weiterbehandlung und der Nutzung der bisherigen Akte zu. Die Aufbewahrungspflicht gilt dann auch für die Altunterlagen.
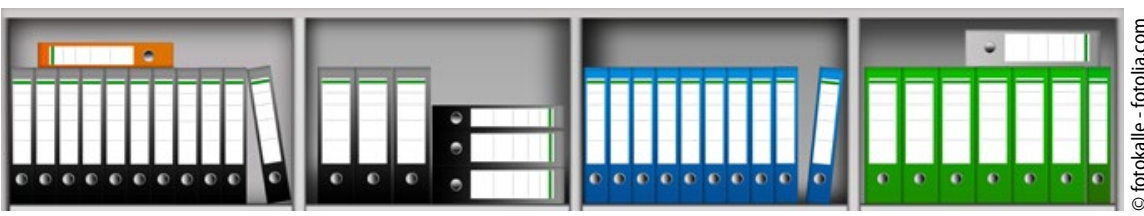

Selbst ordentlich aussehende Papierarchive hat niemand gern im Haus.

Grundsätzlich sollten Sie alles daran setzen, eine physische Ablage zu vermeiden. Dafür kann die Aufbewahrungspflicht auch an den Patienten übergeben werden. Alle Arztbriefe, Befunde etc. werden eingescannt, die Originale werden - gekennzeichnet $\mathrm{z}$. B. mit einem Stempel „Scan“ - dem Patienten ausgehändigt. Dieser bestätigt die Vorgehensweise einmal schriftlich auf einem Formular, das natürlich wiederum gescannt wird. Die Methode ist auch für ältere
Fälle sinnvoll. So werden einige Regalmeter für Altunterlagen gespart.

Kommt man an dem papiernen Übel partout nicht vorbei, empfiehlt sich eine jahrgangsweise organisierte Lagerung. Dann kann man sich nach Ablauf der Frist Jahr für Jahr eines Teils der Unterlagen entledigen.

Vorsicht: Wenn Sie einen Entsorgungsbetrieb in Anspruch nehmen, muss er zur Vernichtung sensibler Daten zugelassen sein.

\section{Gibt es Haftungsbeschränkung für MVZ-Partner?}

$?$ Dr. K. F., Allgemeinarzt, Hessen: Ich möchte ein MVZ gründen. Gibt es hier die Möglichkeit einer Haftungsbeschränkung wie bei einer $\mathrm{GmbH}$ ?

Antwort: Wir Ärzte müssen bei solchen Fragen auch immer das Heilberufekammergesetz beachten. In Bayern wurde hier 2015 der Zusammenschluss von Ärzten und Psychotherapeuten mit begrenzter persönlicher Haftung im Rahmen einer Partnerschaft mit beschränkter Berufshaftung (PartmbB) ermöglicht. Gemäß $₫ 8$ Abs. 4 S. 1 des Partnerschaftsgesetzes (PartGG) ist dann die Haftung gegenüber Gläubigern für Schäden, die aus fehlerhafter Berufsausübung entstehen, auf das Gesellschaftsvermögen beschränkt. Damit ist eine große Hemmschwelle beseitigt, nämlich der Rückgriff auf das Privatvermögen des einzelnen Partners.
Ausgenommen ist die Berufshaftpflichtversicherung, die auch in einer PartmbB zwingend ist. Hier geht es um hohe Prämien und Risiken, weshalb sich Ärzte von einem versicherungsunabhängigen Versicherungsfachmann beraten lassen sollten.

Ihnen in Hessen kann ich vorerst nur raten, bei der Landesärztekammer zu eruieren, ob und wann man dem bayerischen Vorbild folgen will. 